\title{
Visualization of Carotenoid-Storage Structures in Fruits by Transmission Electron Microscopy
}

Joanna Lado 1

Jaime Zacarias 2

María Jesús Rodrigo 2

Lorenzo Zacarias $2 \square$

Email 1zacarias@iata.csic.es

1 Instituto Nacional de Investigación Agropecuaria (INIA), Salto, Uruguay

2 Instituto de Agroquímica y Tecnología de Alimentos, Consejo Superior de Investigaciones Científicas (IATA-CSIC), Valencia, Spain

\section{Abstract}

Plastids are cell organelles that, beside other functions, have the capability to store carotenoids in specialized structures, which may vary among the different plant species, tissues or according to the carotenoid complement. Fruits are an important source of carotenoids, and during ripening, chloroplasts differentiate into chromoplasts that are able to accumulate large amounts of carotenoids, rendering then the characteristic fruit coloration. Whereas lycopene or $\beta$-carotene may accumulate as crystal in the chromoplasts of some fruit, other xanthophyll-accumulating fruits differentiate plastoglobuli as a preferred system to enhance carotenoids stability and storage. Visualization of plastid ultrastructure and their transformation during ripening or in fruit of contrasting coloration are fundamental objectives within carotenoids research in fruits. Therefore, in this chapter, we describe a protocol for the visualization and analysis of plastid ultrastructure by transmission electron microscopy (TEM), specially designed and adapted to fruit tissues.

Key words

Carotenoids

Citrus fruits

Chloroplast

Chromoplast

Plastids

Plastoglobuli

Transmission electron microscopy

\section{Introduction}

Carotenoids are a group of tetraterpenoid pigments synthetized in the plastids of plants and in some algae, fungi, and bacteria [1]. They are responsible for the attractive coloration of flowers, fruits, and other tissues in many plant species and also play critical roles in human nutrition and health $[2,3,4]$.

Accumulation of carotenoids in plants is the result of a fine balance between biosynthesis, degradation, and their accumulation in specialized structures, the plastid [5]. During plant development and tissue 
differentiation, there are substantial changes in the number of plastids and in their structure and functionality that may determine the capacity for carotenoid storage. Various types of plastids can be identified in plant cells, such as proplastids, amyloplasts, etioplasts, chloroplasts, and chromoplasts [6, 7]. All these types of plastids, except proplastids, possess the capacity for carotenoid production [8, 9]. Amyloplasts are starch-storing plastids found in seed, roots and tubers (wheat, maize, potato, cassava) and in general contain relatively low levels of carotenoids, mainly xanthophylls (i.e., lutein, zeaxanthin, and violaxanthin) $[8,10]$. Etioplasts are developed in dark-grown plants and usually contain small amounts of photosynthetic carotenoids, primarily lutein and violaxanthin, as well as the chlorophyll precursor protochlorophyllide [11]. Chloroplasts are the typical plastids found in green photosynthetic tissues (leaves and immature fruits) while the synthesis of carotenoids is believed to occur in their envelop and in the thylakoid membrane $[12,13,14]$. The formation of a lipoprotein complex between chlorophyll and carotenoids allows the accumulation of large amounts of carotenoids in the chloroplasts. Lutein, $\beta$-carotene, violaxanthin and neoxanthin are the most abundant carotenoids in chloroplast [14]. Finally, chromoplast are specialized plastid with large capacity to synthetize and accumulate massive amounts of carotenoids, and then providing particular coloration to specialized tissue, such as flowers or fruits $[9,15$, $16]$.

Chromoplasts in most tissues contain plastoglobuli, which are lipid body structure accumulating carotenoids. Moreover, other types of carotenoid-accumulating structures may also be found inside the chromoplast, which determine their classification as globular, tubular, fibrillary, crystalline, and membranous $[9,15]$. Globular chromoplasts are characterized by abundant plastoglobule, and are characteristics of many fruits such as mango (Mangifera indica), yellow papaya (Carica papaya), tomato (Solanum lycopersicum) [17], saffron stigma [18] and citrus [19, 20]. Globular chromoplasts usually contain lutein, $\beta$-cryptoxanthin, and $\beta$-carotene as major carotenoids [21]. Crystalline chromoplasts typically accumulate large amount of lycopene and $\beta$-carotene, as red/orange crystals and are abundant in tomato [17] and carrot (Daucus carota) [22]. Interestingly, while all-trans- $\beta$-carotene is deposited as crystalline, its cis isomers are in globular chromoplasts [23]. Membranous chromoplasts are typified by the large amount of condensed multi-layer membrane structures, as shown in orange cauliflower [24]. Fibrillar chromoplasts contain spindle-shaped structures with numerous tubules arranged in bundles, as in yellow papaya [21]. In general, fruits with low carotenoid content do not differentiate plastids of define any structure, whereas fruit accumulating uncommon carotenoids develop uncommon structures. The high heterogeneous nature or plasticity of the carotenoid-sequestering substructures in the chromoplasts is determinant of the diversity and the levels of carotenoids accumulating in different plant tissues.

The diversity in coloration and in the carotenoid content and composition in the peel and pulp of citrus fruits, provide an excellent system for the morphological and structural analysis of plastid diversity into the chromoplast and the relation with the carotenoid complement [25, 26, 27]. Citrus plastids have an extraordinary plasticity to rearrange carotenoids inside different compartments to allow massive pigment accumulation, probably as a mechanism to avoid potential harmful effects [28]. Transmission electron microscopy (TEM) observations of peel and pulp sections of oranges (Fig. 1) and grapefruits (Fig. 2) of contrasting coloration, reveal striking differences in chromoplast structure, potentially related to their particular colors and to their unique carotenoid complement. The peel and pulp of ordinary orange fruit that accumulate high amounts of xanthophylls contained even and typical round plastoglobuli, which are easily visualized as electron-dense bodies of variable shape and size, coexisting in certain cases with starch grains (Fig. $1 \mathrm{a}-\mathrm{c}$ ). The pulp of the yellow orange mutant Pinalate accumulates large amounts of unusual carotenoids, such as phytoene, phytofluene and $\zeta$-carotene, and interestingly, their chromoplasts contain novel even round vesicles, instead of the characteristics plastoglobuli of the ordinary oranges (Fig. 1d). Mature fruit of red grapefruit, such as Star Ruby, accumulate lycopene in the pulp and also in the peel (Fig. 2) [28]. Chromoplasts of these tissues contained characteristic plastoglobuli with electrondense bodies of variable size and number. In these cells, the presence of lycopene is evidenced by the observation of long crystals structures (Fig. 2a, c). In yellow sectors of the peel and in the pulp of the white Marsh grapefruit, that contain very low carotenoid concentration, typical plastoglobuli are merely seen, showing instead lipid grains of variable size and achlorophyllous membranes in the peel (Fig. 2b, d). These observations support the motion that a differential carotenoid complement among plant cells 
determinates the type of plastid substructures for pigments storage [28].

Fig. 1

Image of the ultrastructure of chromoplast from orange (Citrus sinensis) fruit with distinctive coloration. Peel (a) and pulp (c) of the ordinary mature Navel orange fruit. Magnification of a chromoplast from the peel (b). Pulp of the yellow-mutant Pinalate (d). Structures for carotenoid accumulation are indicated with abbreviations: Pg, plastoglobuli; LG, lipid grains; s, starch grains; Vs, round vesicles typical in the mutant Pinalate

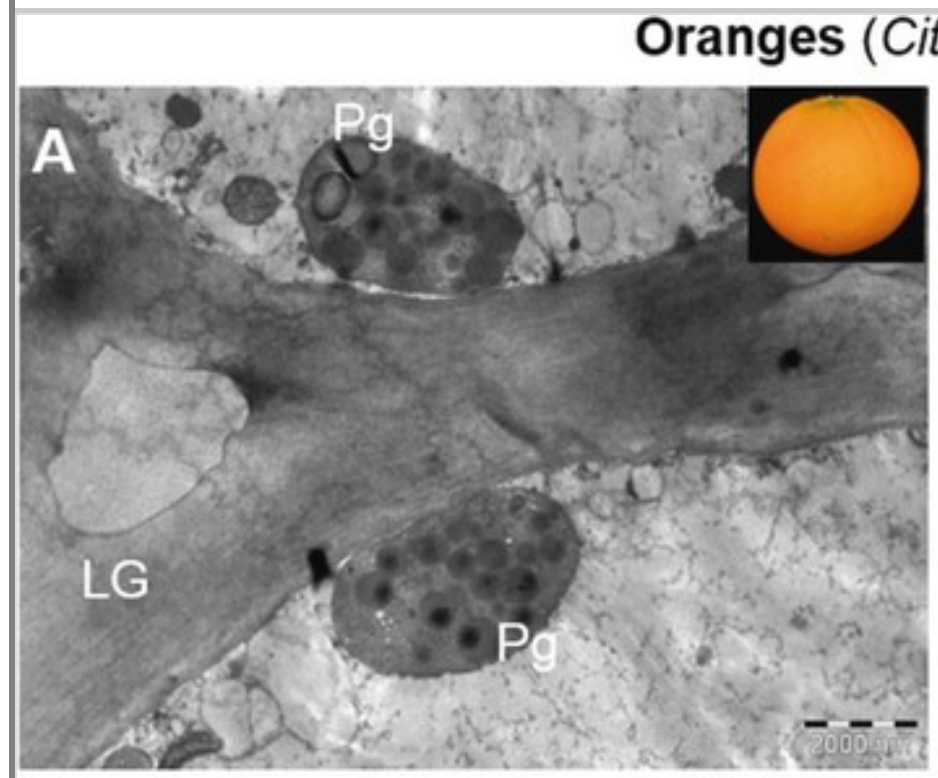

itrus sinensis)
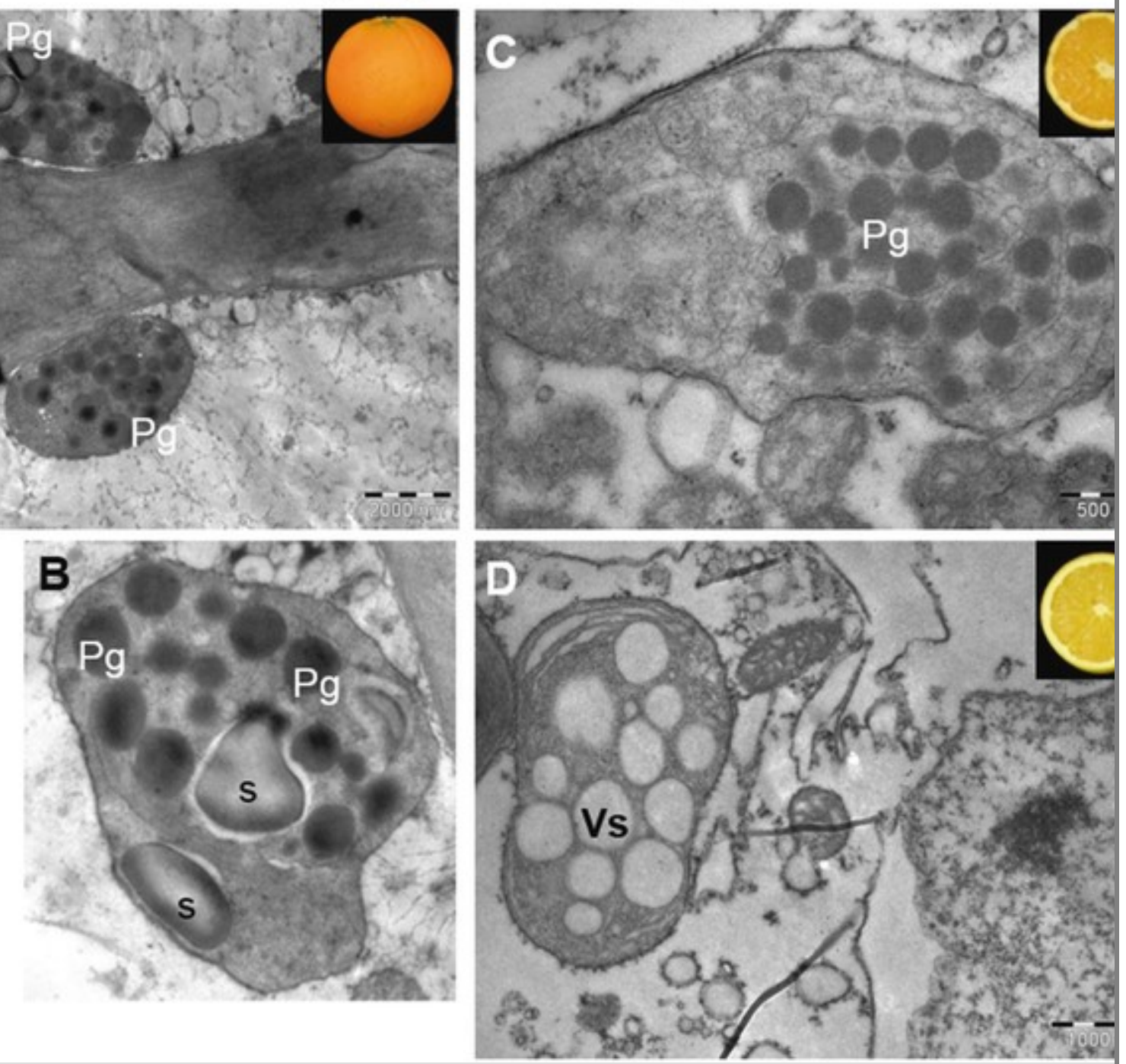
Fig. 2

Image of the ultrastructure of chromoplast from peel and pulp of red and yellow mature grapefruits (Citrus paradisi). Red peel (a) and pulp (c), and yellow peel (b) of Star Ruby grapefruit, and pulp of the yellow Marsh grapefruit (d). The intense red coloration of the peel in Star Ruby grapefruit was achieved by avoiding light exposure of fruit on the field, whereas the yellow fruit was directly exposed to sunlight on the field [19]. Structures for carotenoid accumulation are indicated with abbreviations: Pg, plastoglobuli; LG, lipid grains; s, starch grains; mm, achlorophyllous membranes; c, lycopene crystals with membrane remnants inside (after osmium fixation the crystalloids are largely dissolved during the dehydration procedure and their expanded envelopes are shrunken into an undulating shape)
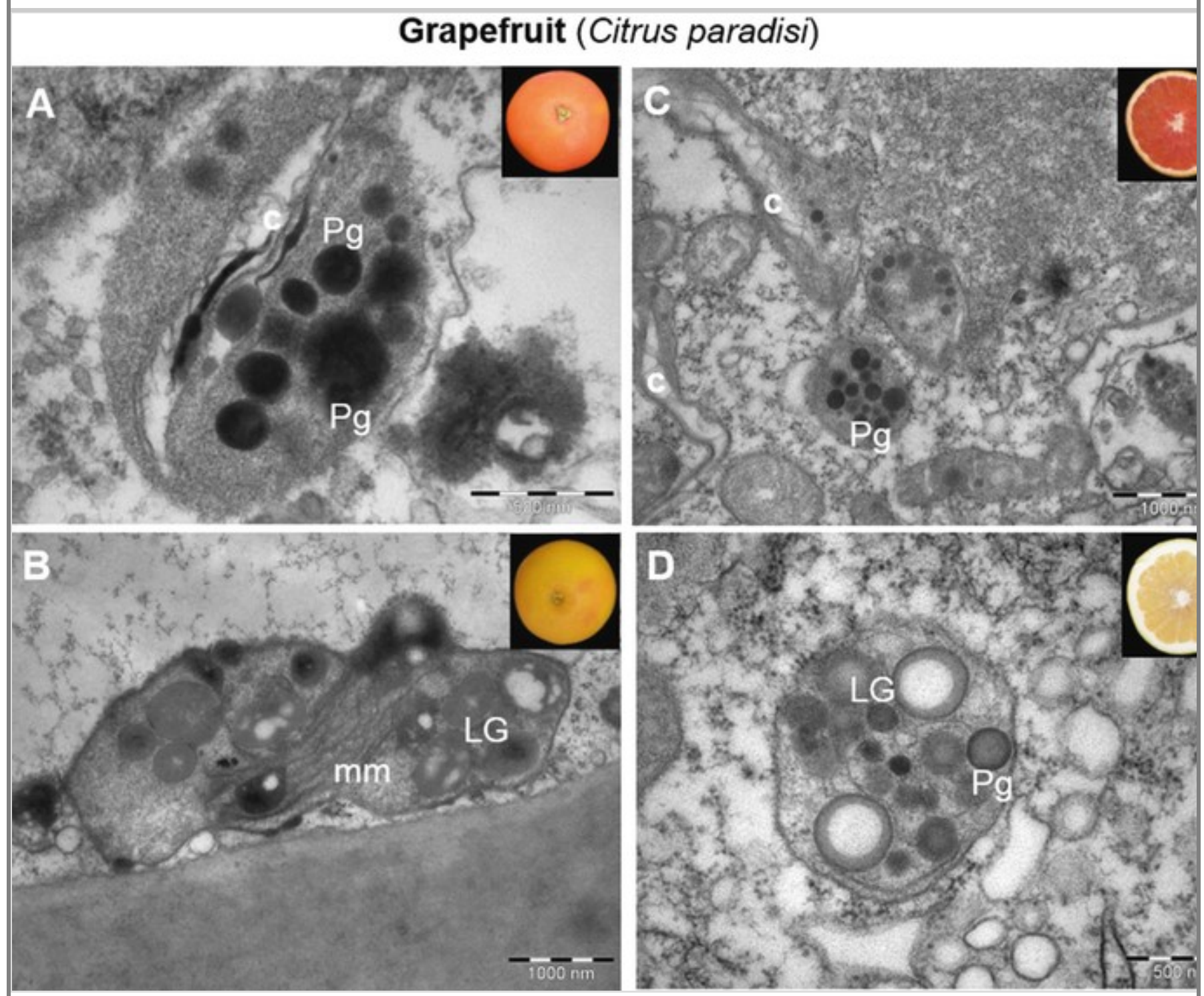

Then, in order to understand the biosynthesis and accumulation of carotenoids in plant cell, and in fruits in particular, it is essential a reliable visualization of the carotenoids-containing structures in cells and plastids ultrastructure. Moreover, carotenoids storage has been also demonstrated to be determinant for their bioaccessibility. In this chapter, we provide a detailed protocol for transmission electron microscopy (TEM) analysis of plastids accumulating carotenoids, specially focused on fruit tissue, since these cells have high potential for carotenoid storage, and exhibit large carotenoid and plastid diversity. 


\section{Materials}

\subsection{Reagents and Solutions}

1. PIPES buffer: Mix piperazine-NN'-bis-2-ethanesulfonic acid (PIPES)- $60.48 \mathrm{~g}$ with $10 \mathrm{M}$ sodium hydroxide $(\mathrm{NaOH})$ to bring the $\mathrm{pH}$ up to 7.2. Make up to $1 \mathrm{~L}$ with distilled water to produce a $0.2 \mathrm{M}$ solution of PIPES buffer. The buffer can be divided up into suitable volumes and frozen until required.

2. Fixative solution ( $4 \%$ formaldehyde): Mix $4 \mathrm{~g}$ of paraformaldehyde with $20 \mathrm{~mL}$ distilled water in a small beaker. Heat to $85^{\circ} \mathrm{C}$ on a hotplate in the fume cupboard. Add three drops of $0.1 \mathrm{M}$ $\mathrm{NaOH}$ and stir until the liquid clears. Make up to $50 \mathrm{~mL}$ with distilled water. This will produce $8 \%$ formaldehyde and it should be then diluted 50/50 in PIPES buffer with glutaraldehyde (see Note 1).

3. Reynolds lead stain: Weight $1.33 \mathrm{~g}$ lead nitrate $\left(\mathrm{Pb}\left(\mathrm{NO}_{3}\right)_{2}\right)$ and $1.76 \mathrm{~g}$ Tri-sodium citrate and place both into $30 \mathrm{~mL}$ distilled water in a very clean glass container. Shake of $1 \mathrm{~min}$ and then intermittently for $30 \mathrm{~min}$ to produce a milky suspension. Add $8 \mathrm{~mL}$ of freshly prepared $1 \mathrm{M}$ sodium hydroxide and shake until clear. Make up to $50 \mathrm{~mL}$ with fresh distilled water. The stain can be divided up into suitable volumes and stored in sealed containers (see Note 2).

4. Uranyl acetate stain: Weight 1-2 g of uranyl acetate (see Note 3) and place it in a centrifuge tube capable of withstanding repeated centrifugation (i.e. polypropylene). Fill the tube with $50 \mathrm{~mL}$ of ethanol $50 \%$ and leave to stand in the fridge overnight. Solution reacts with light forming a precipitate, therefore should be stored in the dark. The tube must be centrifuged before use (see Note 4).

\subsection{Equipment}

1. Scalpels.

2. Petri dishes.

3. Tubes and racks for sample preparation.

4. Microcentrifuge.

5. Nickel grids for TEM samples.

6. Microtome.

7. Glass or diamond knife.

8. Transmission electron microscope (TEM) Hitachi H7000, equipped with a SIS Megaview III digital camera.

\section{Methods}

\subsection{Sample Fixation, Preparation and Inclusion in Resin}


1. The fruit tissue sample should be cut into small squares sections $3-5 \mathrm{~mm}$ long and $1 \mathrm{~mm}$ wide

2. Rinse the sample with buffer (PIPES 0.1 M) for $10 \mathrm{~min}$.

3. Postfix in buffered $1 \%$ osmium tetroxide prepared in 0.1 M PIPES buffer. This step should not exceed more than 1 hour. The sample could not become black in color. Move/rotate the sample during the treatment.

4. Rinse the section with buffer (PIPES 0.1 M) $10 \mathrm{~min}$.

5. Rinse the section briefly in water.

6. Place the fruit section in $2 \%$ uranyl acetate for $20 \mathrm{~min}$ (not longer than $30 \mathrm{~min}$ ) and move/rotate the sections during the treatment. Uranyl acetate staining could be done at this step of afterward (see Subheading 3.3).

7. Place the tissue for dehydration sequentially in the following solutions: ethanol $30 \%$ for $10 \mathrm{~min}$, ethanol $50 \%$ for $10 \mathrm{~min}$, ethanol $70 \%$ for $10 \mathrm{~min}$, ethanol $95 \%$ for $10 \mathrm{~min}$, absolute ethanol for $20 \mathrm{~min}$.

8. Place the section on acetonitrile for $10 \mathrm{~min}$.

9. Place the section in $1 \mathrm{~mL}$ acetonitrile-Spurr resin (50:50) overnight.

10. Place the fruit section in the spur resin solution in continuous rotation during $6 \mathrm{~h}$.

11. Embedding the fruit section in fresh epoxy resin (Spurr) allowing to polymerize in oven at $60{ }^{\circ} \mathrm{C}$ for a period between 20 and $24 \mathrm{~h}$.

12. Collect the rising blocks and store them property labelled in dry environment.

\subsection{Cutting Procedure}

A microtome should be used; a diamond knife is highly recommended, but a glass knife could be also useful, which may be replaced after 2 or 3 cuttings.

1. Place the sample in the correct orientation.

2. Prepare and cut the resin end with a knife or scalpel.

3. Put water in the knife collector/recipient in order to allow the thin sections to launch on the water surface and to be easily recovered.

4. The thin sections could be first observed by optic microscopy in order to guarantee preservation of the tissue structure and quality.

5. A chloroform drop should be included in the water for section stretching before picking.

6. Fish sections with an instrument containing the selected grid. Tissue to be observed should be 
localized in the center of the grid. Sections should be put in nickel grids (and not copper).

7. Leave the sections to dry for $24 \mathrm{~h}$ before staining.

\subsection{Grid Staining}

1. Place a piece of wax-paper into a petri dish.

2. Invert grids section side down onto drops of uranyl acetate solution for $15 \mathrm{~min}$ in the dark (see Notes 5 and 6).

3. Wash gently in a stream of distilled water and blot dry.

4. Invert grids section side down onto drops of Reynolds lead stain $(5 \mathrm{~min})$ in the presence of $\mathrm{NaOH}$ pellets (in order to absorb $\mathrm{CO}_{2}$ ). A petri dish with cover is a suitable place to avoid gas exchange. Lead stain produces a precipitate on exposure to carbon dioxide. Be careful and avoid breathing into samples during staining (see Note 7).

5. Wash gently in a stream of distilled water and blot dry (see Note 8 and 9).

6. Store the staining grids in appropriate racks, properly identified, until microscopic observation (see Note 9).

\subsection{TEM Observations}

1. To initiate TEM observation of the different grids, the microscope should be property adjust following the Microscopy Unit instructions and working protocols that may vary for each particular microscope.

2. As a general procedure, the grid should be mounted over the sample holder, properly adjust to the microscope allowing then perform the vacuum.

3. Selection of appropriate voltage and brightness, and searching for observation cell sections of interest usually at low magnifications to get a wider image of the sections. Increase magnification for an in deep observation discrete of cell sections and organelles ultrastructure.

4. Take pictures of the sections of interest using a SIS Megaview III digital camera.

\section{Notes}

\section{AQ1}

1. Paraformaldehyde and formaldehyde are toxic by inhalation, ingestion and skin absorption and corrosive on skin contact. Wear gloves and use a cupboard.

2. It should be protected from $\mathrm{CO}_{2}$ since produces a precipitate. It should not be used if it becomes cloudy.

3. Lead nitrate is toxic by inhalation and harmful by ingestion and skin absorption. Avoid breathing and use gloves. 
4. Uranyl acetate is very toxic by ingestion and inhalation. Wear gloves and prepare the solution in a fume cup-board.

5. Centrifuge both stains before using (put an aliquot $0.5 \mathrm{~mL}$ in an Eppendorf) and centrifuge at maximum speed.

6. Uranyl acetate should be made always before lead staining.

7. Uranyl acetate produce an undesired precipitate on exposure to light, therefore grids should be stained and stored in darkness afterward.

8. Both stains are harmful by inhalation and ingestion. Avoid breathing dust and wear gloves. In case of spillage wash with abundant water.

9. Leave samples to dry $24 \mathrm{~h}$ before TEM visualization. Store in the dark.

\section{Acknowledgments}

Financial support by research grants AGL-2015-70218 (Ministerio Economía y Competitividad (Spain), and FEDEGENT/2018/007 (Generalitat Valenciana, Spain) are gratefully acknowledged. MJR and LZ are members of Eurocaroten (COST_Action CA15136) and CaRed (Spanish Carotenoid Network, BIO2015-71703-REDT and BIO-2017-90877-REDT).

\section{References}

1. Rodriguez-Concepcion M, Avalos J, Bonet ML, Boronat A, Gomez-Gomez L, Hornero-Mendez D, Limon MC, Meléndez-Martínez AJ, Olmedilla-Alonso B, Palou A, Ribot J, Rodrigo MJ, Zacarias L, Changfu Z (2018) A global perspective on carotenoids: metabolism, biotechnology, and benefits for nutrition and health. Prog Lipid Res 70:62-93

2. Fasset RG, Coombes JS (2012) Astaxanthin in cardiovascular health and disease. Molecules $17: 2030-2048$

3. Mordente A, Guantario B, Meucci E, Silvestrini A, Lombardi E, Martorana GE (2011) Lycopene and cardiovascular diseases: an update. Curr Med Chem 18:1146-1163

4. Rao AV, Rao LG (2007) Carotenoids and human health. Pharmacol Res 55:207-216

5. Li L, Yuan H (2013) Chromoplast biogenesis and carotenoid accumulation. Arch Biochem Biophys 539:102-109

6. Lopez-Juez E, Pyke KA (2004) Plastids unleashed: their development and their integration in plant development. Int J Dev Biol 49:557-577

7. Jarvis P, Lopez-Juez E (2013) Biogenesis and homeostasis of chloroplasts and other plastids. Nat Rev Mol Cell Biol 14:787-802

8. Howitt CA, Pogson BJ (2006) Carotenoid accumulation and function in seeds and non-green tissues. Plant Cell Environ 29:435-445

9. Li L, Yuan H, Zeng Y, Qiang X (2016) Carotenoids in nature biosynthesis, regulation and function. In: Stange C (ed) Plastids and carotenoids accumulation, 1st edn, Switzerland, pp 273-293 
10. Wurtzel E, Cuttriss A, Vallabhaneni R (2012) Maize provitamin a carotenoids, current resources, and future metabolic engineering challenges. Front Plant Sci 3:29

11. Rodriguez-Villalon A, Gas E, Rodriguez-Concepcion M (2009) Phytoene synthase activity controls the biosynthesis of carotenoids and the supply of their metabolic precursors in dark-grown Arabidopsis seedlings. Plant J 60:424-435

12. Niyogi KK, Truong TB (2013) Evolution of flexible nonphotochemical quenching mechanisms that regulate light harvesting in oxygenic photosynthesis. Curr Opin Plant Biol 16:307-314

13. Ruban AV (2016) Nonphotochemical chlorophyll fluorescence quenching: mechanism and effectiveness in protecting plants from photodamage. Plant Physiol 170:1903-1916

14. Rodriguez-Concepcion M, Ruiz-Sola MA (2012) Carotenoid biosynthesis in Arabidopsis: a colorful pathway. Arabidopsis Book 10:e158

15. Egea I, Barsan C, Bian W, Purgatto E, Latche A, Chervin C, Bouzayen M, Pech JC (2010) Chromoplast differentiation: current status and perspectives. Plant Cell Physiol 51:1601-1611

16. Lado J, Zacarias L, Rodrigo MJ (2016) Carotenoids in nature biosynthesis, regulation and function. In: Stange C (ed) Regulation of carotenoid biosynthesis during fruit development, 1st edn, Switzerland, pp 161-198

17. Jeffery J, Holzenburg A, King S (2012) Physical barriers to carotenoid bioaccessibility. Ultrastructure survey of chromoplast and cell wall morphology in nine carotenoid-containing fruits and vegetables. J Sci Food Agric 92:2594-2602

18. Gómez-Gómez L, Parra-Vega A, Rivas-Sendra JM, Seguí-Simarro R, Molina V, Pallotti C, RubioMoraga A, Diretto G, Prieto A, Ahrazem O (2017) Unraveling massive crocins transport and accumulation through proteome and microscopy tools during the development of saffron stigma. Int $\mathbf{J}$ Mol Sci 18:76

19. Lado J, Cronje P, Alquezar B, Page A, Manzi M, Gomez-Cadenas A, Stead AD, Zacarias L, Rodrigo MJ (2015) Fruit shading enhances peel color, carotenes accumulation and chromoplast differentiation in red grapefruit. Physiol Plant 154:469-484

20. Lu P, Wang C, Yin T, Zhong S, Grierson D, Chen K, Xu C (2017) Cytological and molecular characterization of carotenoid accumulation in normal and high-lycopene mutant oranges. Sci Rep $7: 761$

21. Schweiggert RM, Steingass CB, Heller A, Esquivel P, Carle R (2011) Characterization of chromoplasts and carotenoids of redand yellow-fleshed papaya (Carica papaya L.). Planta 234:1031-1044

22. Kim JE, Rensing KH, Douglas CJ, Cheng KM (2010) Chromoplasts ultrastructure and estimated carotene content in root secondary phloem of different carrot varieties. Planta 231:549-558

23. Vasquez-Caicedo AL, Heller A, Neidhart S, Carle R (2006) Chromoplast morphology and betacarotene accumulation during postharvest ripening of mango cv. 'Tommy Atkins'. J Agric Food Chem 54:5769-5776

24. Paolillo DJ, Garvin DF, Parthasarathy MV (2004) The chromoplasts of or mutants of cauliflower (Brassica oleracea L. var. botrytis). Protoplasma 224:245-253 
25. Gross J (1987) Pigments in fruits. In: Schweigert BS (ed) Food science and technology: a series of monographs. Academic Press. London Sci Technol

26. Alquézar B, Rodrigo MJ, Zacarías L (2008) Regulation of carotenoid biosynthesis during fruit maturation in the red-fleshed orange mutant Cara Cara. Phytochemistry 69:1997-2007

27. Rodrigo MJ, Alquézar B, Alos E, Lado J, Zacarias L (2013) Biochemical bases and molecular regulation of pigmentation in the peel of Citrus fruit. Sci Hortic 163:46-62

28. Lado J, Zacarías L, Gurrea L, Page A, Stead A, Rodrigo MJ (2015) Exploring the diversity in Citrus fruit colouration to decipher the relationship between plastid ultrastructure and carotenoid composition. Planta 242:645-661 